\title{
State Estimation of Distribution Network Considering Data Compatibility
}

\author{
Shengtao Wu, Yan Li \\ School of Electrical and Electronic Engineering, Huazhong University of Science and Technology, Wuhan, China \\ Email: 1280251926@qq.com
}

How to cite this paper: $\mathrm{Wu}, \mathrm{S}$.T. and $\mathrm{Li}, \mathrm{Y}$. (2020) State Estimation of Distribution Network Considering Data Compatibility. Energy and Power Engineering, 12, 73-83. https://doi.org/10.4236/epe.2020.124B008

Received: March 12, 2020

Accepted: April 7, 2020

Published: April 10, 2020

\begin{abstract}
Considering that the measurement devices of the distribution network are becoming more and more abundant, on the basis of the traditional Supervisory Control And Data Acquisition (SCADA) measurement system, Phasor measurement unit (PMU) devices are also gradually applied to the distribution network. So when estimating the state of the distribution network, the above two devices need to be used. However, because the data of different measurement systems are different, it is necessary to balance this difference so that the data of different systems can be compatible to achieve the purpose of effective utilization of the estimated power distribution state. To this end, this paper starts with three aspects of data accuracy of the two measurement systems, data time section and data refresh frequency to eliminate the differences between system data, and then considers the actual situation of the three-phase asymmetry of the distribution network. The three-phase state estimation equations are constructed by the branch current method, and finally the state estimation results are solved by the weighted least square method.
\end{abstract}

\section{Keywords}

Distribution Network, State Estimation, Data Compatibility, Branch Current Method

\section{Introduction}

The state estimation algorithm mainly improves the accuracy of the state estimation by increasing the redundancy of the data, so the state estimation is also called filtering [1]. Traditional state estimation is mainly used in transmission grids, and there are relatively few applications in distribution networks. Until recent years, the state estimation of distribution networks has been widely concerned by researchers. However, the distribution network is different from the 
transmission network and has its own characteristics, such as three-phase asymmetry. Therefore, the state estimation of the transmission system is not suitable for the distribution network [2]. Therefore, it is necessary for us to conduct targeted research on the state estimation of the power distribution system.

With the development of distribution automation technology, measurement devices for distribution networks have gradually increased, and monitoring of distribution systems has become more and more detailed. The types of distribution network measurement devices are also getting richer. On the basis of the SCADA measurement system, PMU devices have also appeared in the distribution network, and the data accuracy has improved. In reference [3], the author proposed a state estimation method for distribution network systems with high-precision PMU measurement data, but the establishment of the method did not specify the specific details of its construction. In reference [4], the author also highlights the role of high-precision PMU data for state estimation, and analyzes the advantages and disadvantages of state estimation in polar and rectangular coordinate systems. The results show that the accuracy of distribution network state estimation is improved. In general, it is better to choose a rectangular coordinate system for solving. In reference [5], the author believes that there will be a PMU measurement system in the distribution network in the future. Using this high-precision system, the error of the state variables is minimized by optimizing the scheme to achieve the purpose of improving the estimation accuracy.

However, there are many differences in the data of these two systems. Generally, the SCADA data has insufficient accuracy, no time scale, and the data refresh frequency is low. The PMU data has high accuracy, but the number of installations in the distribution network is relatively small but the data frequency is refreshed rate is higher. If it is not processed, the effect of the distribution network state estimation will be greatly reduced. However, there is relatively little research in this area. In reference [6], the author considers the problem of inconsistent time sections in the transmission process of different measuring devices. Therefore, the time delay error is used to compensate for the error caused by the communication delay, and the problem caused by the inconsistent time section is improved. The problems brought by it ultimately improve the accuracy of state estimation. In reference [7], the author used the curve fitting method to make up for the data of the measurement system with low data refresh frequency, but the data accuracy needs to be improved. In reference [8], the author mainly considered the impact of PMU data on the accuracy of state estimation, but did not mention the data difference between different systems. In reference [9], the author considered the fusion of PMU data and SCADA data, and used a hybrid state estimation algorithm to solve it, but for the problem of inconsistent time sections, no effective solution was proposed.

Based on the above research, this article first analyzes the different characteristics of SCADA and PMU data, and then based on the specific differences be- 
tween them, gives corresponding solutions from three aspects: data accuracy, time section, and data refresh frequency. Then, based on the three-phase line model of the distribution network, the branch current method is used to estimate the state of the distribution network considering data compatibility.

\section{Difference Analysis between SCADA Data and PMU Data}

So far, medium voltage distribution feeders lack the necessary infrastructure to ensure the observability of the network [10]. Considering the economics of power grid deployment facilities, multiple measurement systems need to cooperate to provide measurement data. The two main devices in the system are introduced and compared below.

The PMU measures the DC, switching, phase-detection pulse, terminal voltage, terminal current, and three-phase current, three-phase voltage, and switching signals of the unit in real time. Then, the PMU calculates the measured signals to obtain the internal potential, speed, and electrical quantities such as active power, reactive power, and frequency of the line, and can perform primary / secondary value conversion. The real-time output rate of the PMU is in the millisecond level, and some literatures specifically record that data is transmitted once every $10 \mathrm{~ms}$, which can be selected according to the master station.

SCADA systems collect data at HV-MV substations, typically at rates ranging from a few seconds to a minute. For radial feeders, this measurement usually includes the medium-voltage bus voltage amplitude and bus current, assuming the load has an average power factor, so that the total $\mathrm{P}$ and total $\mathrm{Q}$ delivered by the feeder can be calculated. So far, this is actually the only telemetry information at the medium voltage level for most utilities [11].

In Table 1, it compares these two measurements. It can be seen that the PMU data has higher accuracy and time stamping, while the SCADA data has lower accuracy and no time stamping, and the refresh frequency is lower than that of PMU. Therefore, when performing state estimation, a compatibility analysis of these two types of data is required.

\section{Analysis of Two Types of Data Compatibility}

\subsection{Analysis of Data Precision Compatible Methods}

Since the accuracy of the three-phase data of the SCADA system is lower than that of the three-phase data of the PMU system, in a medium-voltage distribution

Table 1. Comparison of SCADA and PMU data.

\begin{tabular}{|c|c|c|c|c|}
\hline $\begin{array}{l}\text { Measurement } \\
\text { system }\end{array}$ & Measurement data & Refresh rate & Accuracy class & Time stamp \\
\hline SCADA & $\begin{array}{c}\text { Three-phase voltage, three-phase } \\
\text { current, three-phase power }\end{array}$ & Second level & Lower & No \\
\hline PMU & $\begin{array}{l}\text { Three-phase voltage phasor, } \\
\text { three-phase current phasor }\end{array}$ & $\begin{array}{l}\text { Millisecond } \\
\text { level }\end{array}$ & high & Yes \\
\hline
\end{tabular}


network that mixes two types of system data, if they cannot be distinguished well, it may be an improvement for the medium-voltage distribution network The accuracy of state estimation will not help much. At present, a common method is to use the error distribution of each system as the weight of the system data. Then high-precision data will naturally have a high weight coefficient, and low-precision data will have a lower weight. This article will not go into too much detail.

\subsection{Analysis of Data Time Section Compatibility Methods}

Because the three-phase data of the SCADA system does not have a time stamp, the three-phase data of the PMU system has a time stamp. Then, in the state estimation of the distribution network, because the time scale of the SCADA three-phase data cannot be accurately understood, this will cause the state estimation to be significantly different from the actual state of the system, and therefore will have a greater impact on the accuracy of the state estimation.

Based on this, this article considers that the upload frequency of the PMU is milliseconds, and assumes that the specific upload rate is set to $10 \mathrm{~ms}$. Under normal circumstances, this article believes that within a $10 \mathrm{~ms}$ time interval, the possibility of a large change in the related electricity parameters is small or even negligible. Therefore, in order to add timestamps to the relevant SCADA three-phase data, and also to ensure that the SCADA three-phase data and the PMU three-phase data can be at the same time section, this article will introduce the introduction of buffers in the computer and define the concept of time buffers. That is, by setting a time buffer with a time interval of $10 \mathrm{~ms}$ at the time of PMU three-phase data upload, and thinking that as long as there is relevant SCADA three-phase data upload in this time zone, the time scale of the SCADA three-phase data is related to PMUs have the same time scale. This not only adds time scales to the SCADA three-phase data, but also unifies the PMU three-phase data and SCADA three-phase data on the same time section, which facilitates the subsequent state estimation of the distribution network.

As shown in Figure 1, it is assumed that the three-phase data of the PMU is uploaded at 10 milliseconds. At this time, the three-phase data of the SCADA is not uploaded, but is slightly later than the PMU, and also in the time buffer

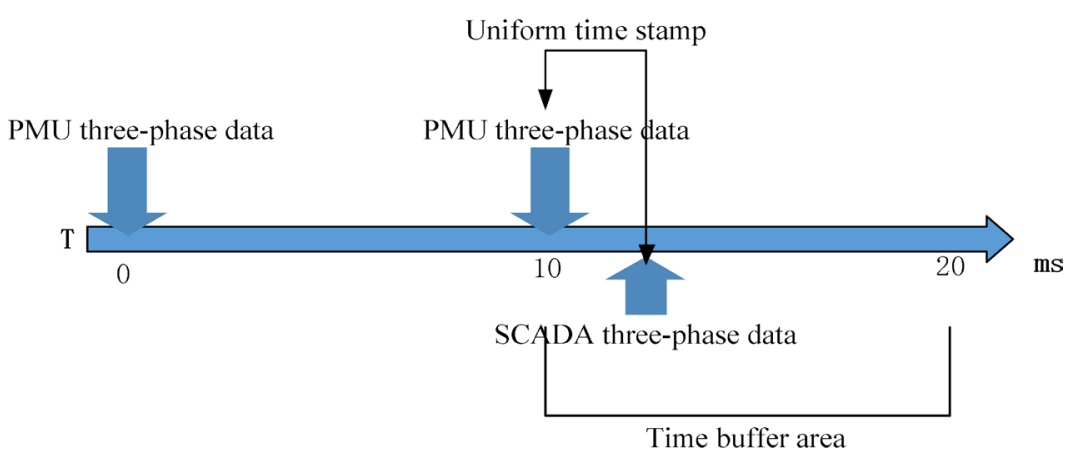

Figure 1. Adding SCADA three-phase data time stamp to unify time sections. 
based on the PMU at this moment. This article considers that the upload time of SCADA data uploaded at this time is at 10 milliseconds.

\subsection{Analysis of Data Upload Frequency Compatible Methods}

On the issue of different upload frequencies. First, this article understands that the upload frequency of the three-phase data of the SCADA system is on the second level, which is assumed to be 2 seconds, and the upload frequency of the three-phase data of the PMU system is on the millisecond level, which is also assumed to be $10 \mathrm{~ms}$ here. In this way, we can know that in the same medium-voltage power distribution system, the PMU has been uploaded dozens of times by the SCADA system in the time interval for uploading data once. If they cannot be fully utilized, this will waste a lot of high precision data. The PMU data is also a huge data loss for the state estimation, which makes the system dispatcher unable to grasp the system state information more accurately.

For this reason, this article also considers this problem under normal circumstances, that is, the change of power data in a short period of time is considered stable. For example, at the second or millisecond level, we can treat the data change during this period as approximately linear, then we can use the linear change rule to handle the problem of inconsistent upload frequency.

Based on the above analysis, this paper intends to use Lagrangian low-order linear interpolation algorithm to solve the problem of frequency compatibility of three-phase data in different systems, and in the following description of this article, this method will be referred to as the interpolation algorithm for short. Because this method is divided into interpolation algorithm and extrapolation algorithm, and the interpolation algorithm has a better effect on the internal interpolation of the data, the extrapolation method is mainly aimed at the external interpolation of the data. Therefore, this paper will use the interpolation algorithm to enrich the historical database for frequency compatibility of historical data; while the frequency compatibility of current or future time section data will be extrapolated.

Regarding frequency compatible interpolation methods. Regarding frequency compatible interpolation methods. As shown in Figure 2(a) below, as the interpolation interval decreases from $\mathrm{T} 2$ to $\mathrm{T} 1$, the interpolation result changes from $\mathrm{X} 2$ to $\mathrm{X} 1$, and it can be seen that the accuracy also increases. So if you choose a larger interpolation interval, the interpolation accuracy will be unsatisfactory, and the minimum upload frequency of SCADA data is 2 seconds. Therefore, for SCADA data frequency to match the data of PMU, the interpolation time interval used by its interpolation algorithm can be 2 seconds. Then the calculation formula for the phasor measurement of a specific SCADA node is shown in the following Equation (1) and this paper considers that this size interval is also used for the extrapolation algorithm, which is also feasible for improving the accuracy.

$$
M_{S C A D A}^{p, T_{\text {insert }}}=M_{S C A D A}^{p, T_{\text {zone1 }}} \frac{T_{\text {insert }}-T_{\text {zone2 } 2}}{T_{\text {zone1 } 1}-T_{\text {zone2 } 2}}+M_{S C A D A}^{p, T_{\text {zone }}} \frac{T_{\text {insert }}-T_{\text {zone1 }}}{T_{\text {zone } 2}-T_{\text {zone } 1}}
$$




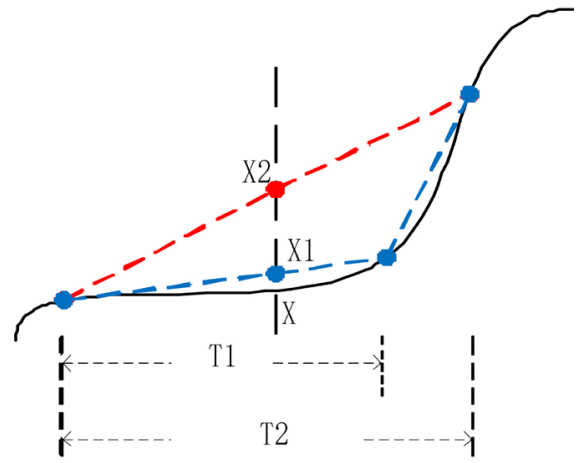

(a)

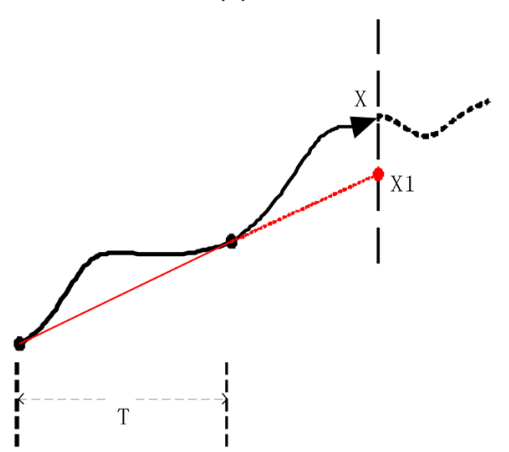

(b)

Figure 2. Frequency compatible interpolation algorithm. (a) Example of interpolation algorithm; (b) Examples of extrapolation algorithms.

In Equation (1): $p$ is the phase sequence number, $T_{\text {insert }}$ is the target interpolation time point, $T_{\text {Zone } 1}$ is the first time point of the interpolation interval, $T_{\text {Zone2 }}$ is the last time point of the interpolation interval, and $T_{\text {Zone1 }}<T_{\text {insert }}<T_{\text {Zone2 }}$.

About frequency compatible extrapolation algorithms. As shown in Figure 2(b), considering that the data on the current time section is not consistent with the frequency, the SCADA system may not upload new data on this time section. At this time, only the PMU data is not feasible for state estimation. Based on this, in this scenario, this paper uses the extrapolation algorithm to quickly make up for the SCADA measurement data that is not available in this time section, thereby uniformly refreshing the frequency at this angle. At this time, the calculation formula for the interpolation result of the measured value of a certain SCADA configuration point at a specified time is shown in the following Equation (2).

$$
M_{S C A D A}^{p, T_{\text {insert }}}=M_{S C A D A}^{p, T_{\text {insertzone1 }}} \frac{T_{\text {insert }}-T_{\text {insertzone 2 }}}{T_{\text {insertzone1 } 1}-T_{\text {insertzone2 }}}+M_{\text {SCADA }}^{p, T_{\text {insertone } 2}} \frac{T_{\text {insert }}-T_{\text {insertzone1 }}}{T_{\text {insertzone } 2}-T_{\text {insertzone1 } 1}}
$$

In Equation (2): $T_{\text {interzone }}$ is the first time point of the interpolation interval, $T_{\text {interzone2 } 2}$ is the last time point of the interpolation interval, and

$T_{\text {interZone1 }}<T_{\text {interZone2 }}<T_{\text {insert }}$.

\section{State Estimation Analysis}

Because the three-phase asymmetry factor must be considered in the state esti- 
mation of the distribution network, this article focuses on modeling the three-phase line parameters of the distribution network before the state estimation of the distribution network. The three-phase model of the specific line is shown in Figure 3.

Among them, $Z_{a a}, Z_{b b}$, and $Z_{c c}$ are the self-impedance of the three-phase line; $Z_{a b}, Z_{b c}$ and $Z_{c a}$ are the mutual impedance of the three-phase line; $I_{a}, I_{b}$, and $I_{c}$ are the currents flowing through the three-phase line.

The branch impedance matrix of a three-phase line is shown in Equation (3) below:

$$
Z_{a b c}=\left(\begin{array}{lll}
Z_{a a} & Z_{a b} & Z_{c a} \\
Z_{a b} & Z_{b b} & Z_{b a} \\
Z_{c a} & Z_{b a} & Z_{c c}
\end{array}\right)
$$

The state estimation in this paper is to use the branch current method to establish the related estimation equation. That is to say, the state quantity selected for the state estimation is the branch current amplitude and phase angle. At the same time, considering the three-phase state, the state variable shape is shown in the following Equation (4):

$$
\left(I_{1,2}^{a}, I_{1,2}^{b}, I_{1,2}^{c}, \beta_{1,2}^{a}, \beta_{1,2}^{b}, \beta_{1,2}^{c}, \cdots, I_{i j}^{a}, I_{i j}^{b}, I_{i j}^{c}, \beta_{i j}^{a}, \beta_{i j}^{b}, \beta_{i j}^{c}, \cdots\right)
$$

In Equation (4): $I_{i j}^{a}$ is the branch a-phase current of the first node $i$, and the end node is $j ; \beta_{i j}^{a}$ represents the phase angle of the corresponding branch current. By the same token, the meanings of the other two related symbols can be obtained.

In addition, because this article is for the state estimation of the distribution network with SCADA system and PMU system, the data of the two different systems are compatible, so pay attention to this compatibility process when performing state estimation.

Then, in the state estimation of the distribution network, when the SCADA system data and the PMU system data are simultaneously transmitted to the state estimation data system, the state estimation process is directly performed. Otherwise, the interpolation algorithm is used for making up the SCADA data, and then the state estimation is performed.

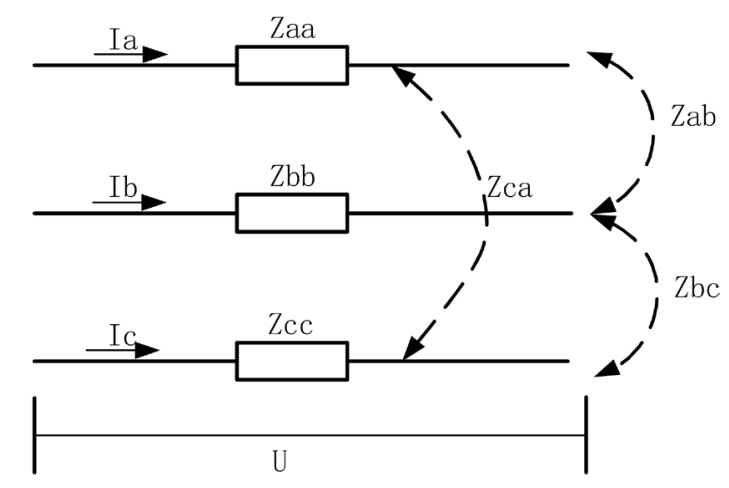

Figure 3. Three-phase line model of distribution network. 
In summary, the measurement equation matrix for state estimation in this paper is shown in Equation (5) below:

$\left(\begin{array}{c}P_{i j,(P M U, S C A D A)}^{a} \\ P_{i j,(P M U, S C A D A)}^{b} \\ P_{i j,(P M U, S C A D A)}^{c} \\ P_{i,(P M U, S C A D A)}^{a} \\ P_{i,(P M U, S C A D A)}^{b} \\ P_{i,(P M U, S C A D A)}^{c} \\ \vdots \\ Q_{i j,(P M U, S C A D A)}^{a} \\ \vdots \\ Q_{i,(P M U, S C A D A)}^{a} \\ \vdots \\ U_{i,(P M U, S C A D A)}^{a} \\ \vdots \\ I_{i j,(P M U, S C A D A)}^{a} \\ \vdots\end{array}\right)=\left(\begin{array}{c}h_{1}\left(I_{1,2}^{a}, I_{1,2}^{b}, I_{1,2}^{c}, \beta_{1,2}^{a}, \beta_{1,2}^{b}, \beta_{1,2}^{c}, \cdots, I_{i j}^{a}, I_{i j}^{b}, I_{i j}^{c}, \beta_{i j}^{a}, \beta_{i j}^{b}, \beta_{i j}^{c}, \cdots\right) \\ h_{1}\left(I_{1,2}^{a}, I_{1,2}^{b}, I_{1,2}^{c}, \beta_{1,2}^{a}, \beta_{1,2}^{b}, \beta_{1,2}^{c}, \cdots, I_{i j}^{a}, I_{i j}^{b}, I_{i j}^{c}, \beta_{i j}^{a}, \beta_{i j}^{b}, \beta_{i j}^{c}, \cdots\right) \\ h_{1}\left(I_{1,2}^{a}, I_{1,2}^{b}, I_{1,2}^{c}, \beta_{1,2}^{a}, \beta_{1,2}^{b}, \beta_{1,2}^{c}, \cdots, I_{i j}^{a}, I_{i j}^{b}, I_{i j}^{c}, \beta_{i j}^{a}, \beta_{i j}^{b}, \beta_{i j}^{c}, \cdots\right) \\ h_{1}\left(I_{1,2}^{a}, I_{1,2}^{b}, I_{1,2}^{c}, \beta_{1,2}^{a}, \beta_{1,2}^{b}, \beta_{1,2}^{c}, \cdots, I_{i j}^{a}, I_{i j}^{b}, I_{i j}^{c}, \beta_{i j}^{a}, \beta_{i j}^{b}, \beta_{i j}^{c}, \cdots\right) \\ h_{1}\left(I_{1,2}^{a}, I_{1,2}^{b}, I_{1,2}^{c}, \beta_{1,2}^{a}, \beta_{1,2}^{b}, \beta_{1,2}^{c}, \cdots, I_{i j}^{a}, I_{i j}^{b}, I_{i j}^{c}, \beta_{i j}^{a}, \beta_{i j}^{b}, \beta_{i j}^{c}, \cdots\right) \\ h_{1}\left(I_{1,2}^{a}, I_{1,2}^{b}, I_{1,2}^{c}, \beta_{1,2}^{a}, \beta_{1,2}^{b}, \beta_{1,2}^{c}, \cdots, I_{i j}^{a}, I_{i j}^{b}, I_{i j}^{c}, \beta_{i j}^{a}, \beta_{i j}^{b}, \beta_{i j}^{c}, \cdots\right) \\ \vdots \\ h_{1}\left(I_{1,2}^{a}, I_{1,2}^{b}, I_{1,2}^{c}, \beta_{1,2}^{a}, \beta_{1,2}^{b}, \beta_{1,2}^{c}, \cdots, I_{i j}^{a}, I_{i j}^{b}, I_{i j}^{c}, \beta_{i j}^{a}, \beta_{i j}^{b}, \beta_{i j}^{c}, \cdots\right) \\ \vdots \\ h_{1}\left(I_{1,2}^{a}, I_{1,2}^{b}, I_{1,2}^{c}, \beta_{1,2}^{a}, \beta_{1,2}^{b}, \beta_{1,2}^{c}, \cdots, I_{i j}^{a}, I_{i j}^{b}, I_{i j}^{c}, \beta_{i j}^{a}, \beta_{i j}^{b}, \beta_{i j}^{c}, \cdots\right) \\ \vdots \\ h_{1}\left(I_{1,2}^{a}, I_{1,2}^{b}, I_{1,2}^{c}, \beta_{1,2}^{a}, \beta_{1,2}^{b}, \beta_{1,2}^{c}, \cdots, I_{i j}^{a}, I_{i j}^{b}, I_{i j}^{c}, \beta_{i j}^{a}, \beta_{i j}^{b}, \beta_{i j}^{c}, \cdots\right) \\ \vdots \\ h_{1}\left(I_{1,2}^{a}, I_{1,2}^{b}, I_{1,2}^{c}, \beta_{1,2}^{a}, \beta_{1,2}^{b}, \beta_{1,2}^{c}, \cdots, I_{i j}^{a}, I_{i j}^{b}, I_{i j}^{c}, \beta_{i j}^{a}, \beta_{i j}^{b}, \beta_{i j}^{c}, \cdots\right) \\ \vdots\end{array}\right)+v$

In Equation (5): $P_{i j,(P M U, S C A D A)}^{a}$ represents PMU or SCADA active power measurement in a-phase of branch, $i$ and $j$ represent the node numbers; $P_{i,(P M U, S C A D A)}^{a}$ represents the a-phase node load measurement; $I_{i j,(P M U, S C A D A)}^{a}$ is the branch current measurement; $U_{i(P M U, S C A D A)}^{a}$ is the node voltage measurement. Similarly, the meaning of other symbols can be obtained.

Finally, the constructed state estimation equations are solved using the weighted least square method.

\section{Example Analysis and Conclusion}

The simulation of the example passed the IEEE33 node system platform, and the method in this paper was verified by using the 50-second power flow calculation method. It is assumed in the simulation that the upload frequency of the PMU is 1 second (actually milliseconds), and the upload frequency of the SCADA data is 10 seconds (actually 2 seconds). In order to understand the interpolation effect in detail, this article sets a node as a SCADA measurement device, and assumes that the load changes and calculates the power flow. The obtained data is used as the full SCADA measurement value (that is, the data under the assumption that SCADA can be measured every second), and then every 10 seconds is used as a piecewise linear simulation interval, and interpolation is used to solve the interpolation. Obtain the simulation results, that is, the effect between the full measurement value and the interpolation simulation is shown in Figure 4. Use extrapolation to solve the interpolation, the fusion effect is shown in Figure 5.

Through analysis and calculation, the average error of the interpolated simulation value is $0.71 \%$, and the average error of the extrapolated simulation value 


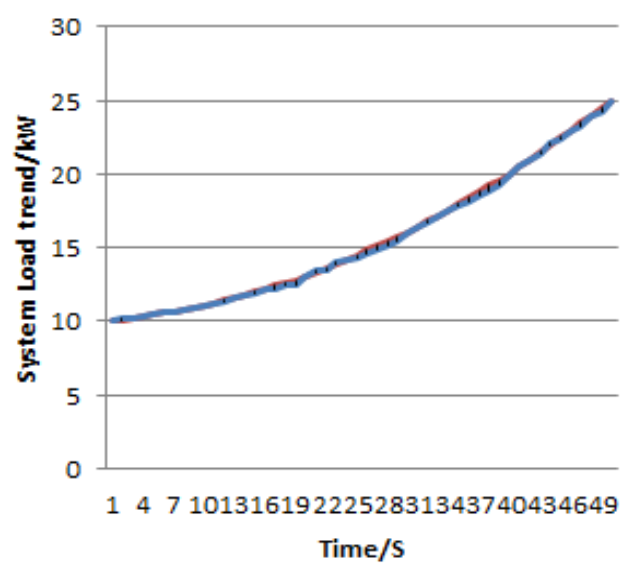

Figure 4. Interpolation simulation analysis.

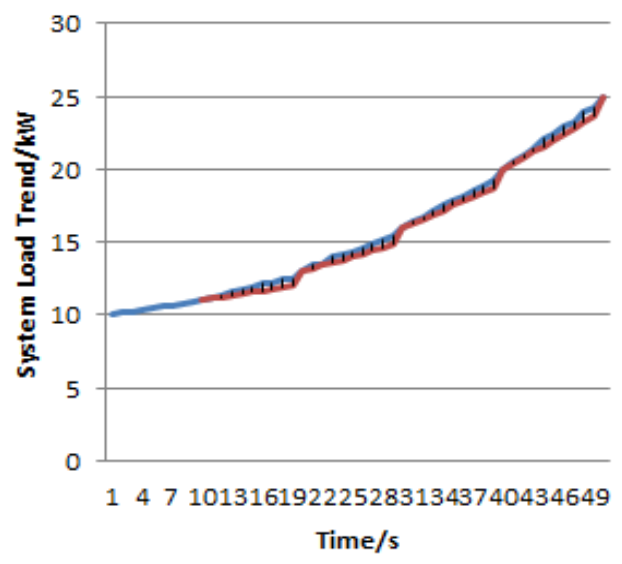

Figure 5. Extrapolation simulation analysis.

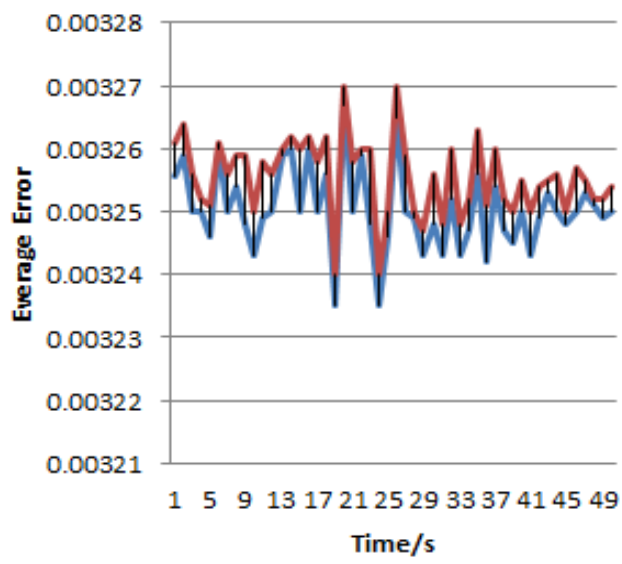

Figure 6. State estimation with data compatibility.

is $1.92 \%$. This paper considers that the error of both methods is acceptable, but the interpolation method is better than the extrapolation method. Therefore, in the calculation process, the solution obtained by the extrapolation method can be corrected by interpolation. 
Use the above results for state estimation analysis. SCADA data is added with a Gaussian error of $2 \%$, and PMU data is added with a Gaussian error of $0.1 \%$. The PMU is configured at nodes $4,6,8,12,17,19,23$, and the remaining nodes are configured with SCADA systems. Compare the effects of full measurement and interpolation, as shown in Figure 6. The results show that the state estimation under interpolation is not much different from the state estimation results under full measurement, which basically meets the requirements. The average error between the two is about $0.1 \%$. The error curves basically match. It shows that the method in this paper can be applied to state estimation.

\section{Acknowledgements}

This work was financially supported by the National key research and development plan: Intelligent Power Network Technology and Equipment (2017YFB0902800).

\section{Conflicts of Interest}

The authors declare no conflicts of interest regarding the publication of this paper.

\section{References}

[1] Wang, K., Mu, G. and Chen, X. (2001) Precision Improvement and Pmu Placement Studies on State Estimation of a Hybrid Measurement System with Pmus. Chinese Society for Electrical Engineering, 21, 29-33.

[2] Van Cutsem, T. and Ribbens-Pavella, M. (1983) Critical Survey of Hierarchical Methods for State Estimation of Electric Power Systems. IEEE Transactions on Power Apparatus and Systems, 102, 3415-3424. https://doi.org/10.1109/TPAS.1983.317838

[3] Alves, G.O., et al. (2016) Distribution System State Estimation Using Phasor Measurement Units. 10 th International Conference on Compatibility, Power Electronics and Power Engineering (CPE-POWERENG), Bydgoszcz, 29 June-1 July 2016, 2166-9546.

[4] Pau, M., Pegoraro, P.A. and Sulis, S. (2013) Efficient Branch-Current-Based Distribution System State Estimation Including Synchronized Measurements. IEEE Transactions on Instrumentation \& Measurement, 62, 2419-2429. https://doi.org/10.1109/TIM.2013.2272397

[5] Liu, J., Ponci, F., Monti, A., Muscas, C., Pegoraro, P.A. and Sulis, S. (2014) Optimal Meter Placement for Robust Measurement Systems in Active Distribution Grids. IEEE Transactions on Instrumentation and Measurement, 63, 1096-1105. https://doi.org/10.1109/TIM.2013.2295657

[6] Alimardani, A., Therrien, F., Atanackovic, D., Jatskevich, J. and Vaahedi, E. (2015) Distribution System State Estimation Based on Nonsynchronized Smart Meters. IEEE Transactions on Smart Grid, 6, 2919-2928. https://doi.org/10.1109/TSG.2015.2429640

[7] Gómez-Expósito, A., Gómez-Quiles, C. and Džafić. I. (2015) State Estimation in Two Time Scales for Smart Distribution Systems. IEEE Transactions on Smart Grid, 6, 421-430. https://doi.org/10.1109/TSG.2014.2335611

[8] Ni, D., Zhang, W., Yu, B. and Gong, W. (2011) A New Algorithm for Power System State Estimation with PMU Measurements. International Conference on Mecha- 
tronic Science, Electric Engineering and Computer, Jilin, 19-22 August 2011, 114-117. https://doi.org/10.1109/MEC.2011.6025413

[9] Saadabadi, H. and Dehghani, M. (2016) Large-Scale Power Systems State Estimation Using PMU and SCADA Data. 2016 24th Iranian Conference on Electrical Engineering (ICEE), Shiraz, 10-12 May 2016, 906-911. https://doi.org/10.1109/IranianCEE.2016.7585649

[10] Primadianto, A. and Lu, C. (2017) A Review on Distribution System State Estimation. IEEE Transactions on Power Systems, 32, 3875-3883. https://doi.org/10.1109/TPWRS.2016.2632156

[11] Li, B. (2013) Research on Dynamic State Estimation of Power System under Mixed Measurement. Ph.D. Thesis, Shanghai Jiaotong University, Shanghai. 